\title{
Dopamine Is a Regulator of Arousal in the Fruit Fly
}

\author{
Kazuhiko Kume, ${ }^{1,2}$ Shoen Kume, ${ }^{1}$ Sang Ki Park, ${ }^{3}$ Jay Hirsh, ${ }^{3}$ and F. Rob Jackson ${ }^{2}$ \\ ${ }^{1}$ Institute of Molecular Embryology and Genetics, Kumamoto University, Kumamoto 860-0811, Japan, ${ }^{2}$ Department of Neuroscience, Center for \\ Neuroscience Research, Tufts University School of Medicine, Boston, Massachusetts 02111, and ${ }^{3}$ Department of Biology, University of Virginia, \\ Charlottesville, Virginia 22904-4328
}

\begin{abstract}
Sleep and arousal are known to be regulated by both homeostatic and circadian processes, but the underlying molecular mechanisms are not well understood. It has been reported that the Drosophila rest/activity cycle has features in common with the mammalian sleep/wake cycle, and it is expected that use of the fly genetic model will facilitate a molecular understanding of sleep and arousal. Here, we report the phenotypic characterization of a Drosophila rest/activity mutant known as fumin $(\mathrm{fmn})$. We show that $\mathrm{fmn}$ mutants have abnormally high levels of activity and reduced rest (sleep); genetic mapping, molecular analyses, and phenotypic rescue experiments demonstrate that these phenotypes result from mutation of the Drosophila dopamine transporter gene. Consistent with the rest phenotype, fmn mutants show enhanced sensitivity to mechanical stimuli and a prolonged arousal once active, indicating a decreased arousal threshold. Strikingly, fmn mutants do not show significant rebound in response to rest deprivation as is typical for wild-type flies, nor do they show decreased life span. These results provide direct evidence that dopaminergic signaling has a critical function in the regulation of insect arousal.
\end{abstract}

Key words: sleep; rest; arousal; Drosophila; dopamine; dopamine transporter

\section{Introduction}

In mammalian species, sleep and wakefulness represent two alternative physiological states, usually defined by the use of electroencephalographic methods. Both circadian and homeostatic processes are known to regulate the cycling between these two states (Borbely, 1982), but the underlying molecular mechanisms and the interactions between the two processes are not well defined. Evidence suggests that neuromodulators, including dopamine (DA), serotonin (5-HT), histamine, norepinephrine, and orexin, as well as small molecules such as adenosine, may function in the regulation of the mammalian sleep/wake cycle (Boutrel and Koob, 2004; Siegel, 2004). In addition, the molecular site of action of wake-promoting substances such as DA receptor agonists (Isaac and Berridge, 2003) and DA uptake inhibitors (Nishino et al., 1998), which act on the dopamine transporter and lead to increased locomotor activity (Wisor et al., 2001), indicate that dopaminergic signaling may be important for the regulation of sleep.

A large amount of evidence indicates that neuromodulators such as 5-HT, octopamine (OA), tyramine (TA), and DA are also

\footnotetext{
Received April 15, 2005; revised June 25, 2005; accepted June 30, 2005.

This work was supported by National Institutes of Health Grants HL59873 (F.R.J.), P30NS047243 (F.R.J.), and GM 27318 (J.H.), National Science Foundation Grant IBN-0234724 (F.R.J.), a grant-in-aid from the Ministry of Education, Culture, Science, Sports and Technology, Japan (K.K., S.K.), and a grant from the Mitshubishi Foundation (S.K.). We thank the Bloomington Stock Center (Indiana University) and Dr. M. Saitoe for fly strains. We are grateful to Drs. A. J. Schroeder, G. P. McNeil, M. Miura, J. D. Levine, and J. Hall for scientific advice and to M. A. Roberts, A. Hamaji, and M. Yamazaki for technical assistance.

Correspondence should be addressed to F. Rob Jackson, Department of Neuroscience, Tufts University School of Medicine, 136 Harrison Avenue, Boston, MA 02111, E-mail: rob.jackson@tufts.edu; or Kazuhiko Kume, Division of Stem Cell Biology, Department of Regenerative Medicine, Institute of Molecular Embryology and Genetics, Kumamoto University, 2-2-1 Honjo, Kumamoto 860-0811, Japan, E-mail: kkume@medic.kumamoto-u.ac.jp.

S. K. Park's present address: Department of Pathology, Harvard Medical School, Boston, MA 02115.

DOI:10.1523/JNEUROSCI.2048-05.2005

Copyright $\odot 2005$ Society for Neuroscience $\quad$ 0270-6474/05/257377-08\$15.00/0
}

important for the regulation of activity in invertebrates including Drosophila (Yellman et al., 1997; Cooper and Neckameyer, 1999; Marder and Bucher, 2001; Pendleton et al., 2002; Banerjee et al., 2004; Dasari and Cooper, 2004; Saraswati et al., 2004; Lima and Miesenbock, 2005). Although these compounds have a well documented role in promoting activity in insects, they have not been extensively investigated in the context of the rest/activity cycle, which has been used as a model for the mammalian sleep/wake cycle by several groups of investigators (Hendricks et al., 2000; Shaw et al., 2000; Ho and Sehgal, 2005). Sleep-like rest states have been described in at least two insects: the cockroach and the fruit fly Drosophila melanogaster (Tobler, 1983; Hendricks et al., 2000; Shaw et al., 2000). In Drosophila, periods of rest, during which flies remain immobile for a few minutes to hours, are behaviorally similar to mammalian sleep. They are (1) consolidated in a circadian manner, (2) regulated by homeostatic mechanisms, and (3) accompanied by a decreased responsiveness to outside stimuli. Additional similarities between mammalian sleep and fly rest behaviors include age-dependent changes in sleep or rest states, pharmacological effects of sleep (rest)-inducing or suppressing drugs such as anti-histaminics and caffeine, and changes in defined molecular markers of sleep (rest)/wake states (Andretic and Shaw, 2005; Ho and Sehgal, 2005). Similar to mammals, it has been suggested that rest may be essential for fly survival, with an effect on life span particularly evident with the cycle circadian mutant $\left(c y c^{01}\right.$ ) (Shaw et al., 2002; Hendricks et al., 2003a).

It seems unlikely that mammalian sleep and Drosophila rest have precisely the same physiological significance. Nonetheless, the use of genetic models is expected to facilitate general insights about the molecular pathways regulating sleep and arousal to the waking state. At present, the similarity of insect rest to mammalian sleep is based primarily on behavioral and pharmacological 
criteria. In this report, we describe a Drosophila hyperactive mutant called fumin ( fmn; meaning sleepless in Japanese) and demonstrate that the behavioral phenotype is caused by a genetic lesion in the dopamine transporter (DAT) gene (Porzgen et al., 2001). The elimination of DAT activity generates a dramatic increase in the length of the active (waking) phase and a corresponding reduction of the inactive phase (sleep or rest), resulting in a nearly sleepless phenotype. The analysis of $f m n$ suggests a role for dopamine in the modulation of insect arousal and highlights a similarity between insects and mammals regarding the molecular basis of arousal.

\section{Materials and Methods}

Fly stocks, crosses, and mutant identification. Flies were reared on a conventional corn meal, yeast, glucose agar medium at $25^{\circ} \mathrm{C}$ as described previously (Newby et al., 1991). Fly stocks used for the recombinational mapping were obtained from the Bloomington Stock Center (Bloomington, IN); the $w^{1118}$ stock used for a backcross is from the M. Saitoe laboratory (Tokyo Metropolitan Institute for Neuroscience, Tokyo, Japan) (Tamura et al., 2003), and all other stocks have been maintained in our laboratory for several years. The $f m n$ mutant was discovered when we isogenized the second chromosome of a $y w$; tim $^{01}$ fly stock for other purposes. One of several separate lines showed exceptionally high locomotor activity compared with the others. fmn was then identified as a second chromosome recessive mutation, which was segregated from tim $^{01}$ by recombination, then identified as a $d D A T$ mutation as described. The fmn mutation was backcrossed with $w$ flies for seven generations to recombinationally separate it from other lesions.

pUAS-dDAT was generated by subcloning of PCR-amplified cDNA containing the dDAT open reading frame (ORF) into the pUAST vector and verified by sequencing. Transgenic flies were generated using standard microinjection procedures. Mutant $(f m n)$ flies transgenic for ELAV-GAL4 (BL-458; $\left.\left.\mathrm{P}^{\{\mathrm{w}+\mathrm{mW} \cdot \mathrm{hs}}=\mathrm{GawB}\right\}^{\text {elav }}{ }^{\mathrm{C} 155}\right)$ and UAS-dDAT were generated by crossing $w$ ELAV-GAL4; fmn to $w$; UAS-dDAT/TM3a, $S b$; fmn flies.

Locomotor activity and rest analysis. Activity was monitored by recording infrared beam crossings of individual flies housed in glass tubes (length, $6.5 \mathrm{~cm}$; inside diameter, $3 \mathrm{~mm}$ ). Events were scored at $1 \mathrm{~min}$ intervals using a Trikinetics (Waltham, MA) Drosophila activity monitoring system (Morales et al., 2002). Unless otherwise specified, 2- to 3 -d-old male flies were used for behavioral analysis, and data were collected continuously for $3 \mathrm{~d}$ under either a $12 \mathrm{~h}$ light/dark (LD) cycle or continuous dark (DD) conditions. The full $3 \mathrm{~d}$ of data were used for quantitative analysis. Data were processed with a Microsoft (Redmond, WA) Excel-based program as described previously (Hendricks et al., 2003a). Five minutes with no activity was defined as a rest bout. The activity index was calculated by dividing total daily activity counts by the length of the active period, which is the total number of minutes during which a fly exhibits at least one activity event. Quantitative analyses were performed with at least 15 male flies (2-5 d after emergence), and the averages with SEMs are displayed. All experiments were replicated at least three times.

Recombinational mapping of $\mathrm{fmn}$. $w$; fmn flies were mated to flies carrying a dominant mutation on the second chromosome (BL-1401; $\left.w g^{\mathrm{Sp}-1} \mathrm{Bl}^{1} \mathrm{~L}^{\mathrm{rm}} \mathrm{Bc} c^{1} \mathrm{Pu}^{2} \mathrm{Pin}^{\mathrm{B}} / \mathrm{SM} 5\right)$, and the female progeny were backcrossed with $w$; fmn males. The resulting $\mathrm{F} 2$ flies with various combinations of dominant phenotypic markers were assayed for activity and used to determine recombination frequencies. About one-half of the flies with either the $L$ (Lobe; cytogenetic locus 51A2-3) or Bc (Black cell; 54F6) marker, but not both, showed the fmn behavioral phenotype, indicating that fmn maps approximately halfway between L and Bc. Subsequently, flies with both the $\mathrm{L}$ and fmn phenotypes were crossed with strains carrying a single P-element insertion at 54B10-13 (BL-11175), 53E6-8 (BL-10931), or 53C9-10 (BL-12056). Female offspring were backcrossed with fmn males. The resulting F2 flies with both the $L$ and the P-element markers were again assayed for the fmn phenotype by monitoring locomotor activity. With BL-11175, BL-10931, and BL-12056, 11 of 47 (23\%), 7 of 41
(17\%), and 1 of $39(3 \%)$ recombinant offspring were phenotypically fmn, indicating fmn maps to the left of and very close to 53C9-10.

Northern blot analysis. Based on the published sequence of dDAT (GenBank accession number, NM_079039), the whole ORF (1896 bp) was PCR amplified from control fly head RNA, cloned into a plasmid vector, and verified by restriction mapping and sequencing. This plasmid was used as template to make both whole ORF and $3^{\prime}$ partial digoxigenin-RNA (DIG-RNA) probes using the SP6 in vitro transcription system (Roche Products, Welwyn Garden City, UK). For the 3' partial probe, RsaI-digested plasmid, which yields a 533 nucleotide probe, was used. For the positive control, a PCR-amplified DIG-DNA probe of rp49 (Vaslet et al., 1980) was used. Total RNAs were prepared from heads of control and fmn flies, using Trizol (Sigma, St. Louis, MO). Poly(A)+ RNA was prepared using an mRNA purification kit (Oligotex-dT; TaKaRa, Tokyo, Japan). Then, $0.7 \mu$ g samples of polyA ${ }^{+}$RNA were fractionated through $1 \%$ agarose, transferred to a nylon membrane, hybridized with the indicated probes, and detected by chemiluminescence.

fmn dDAT gene analysis. PolyA ${ }^{+}$RNA was prepared from fmn fly heads, and the 3' end of dDAT cDNA was PCR amplified, cloned, and sequenced using the GeneRacer kit (Invitrogen, San Diego, CA). Briefly, first-strand cDNA was reverse transcribed using a polydT primer with two general primer sites. Nested PCR was performed first with $5^{\prime}$-ATC CTT CAC CAA CTC GCT GC (dDAT ORF position 410-429) and the $3^{\prime}$ primer combination and next with $5^{\prime}$-CCC TCT TTC CCT ATG CAG TG (dDAT ORF position 805-824) and the $3^{\prime}$ nested primer combination. The resulting PCR product, which had a shorter length than the expected size of dDAT, was cloned and sequenced.

Southern blot analysis was performed using both control and fmn genomic DNA with the entire $\mathrm{DDAT}$ cDNA ORF as a probe. PvuII digestion resulted in a $4.5 \mathrm{~kb}$ band of expected size in control DNA, but a 6.5 $\mathrm{kb}$ band in $f m n$, suggesting that the insertion is $\sim 2 \mathrm{~kb}$ in length.

Mechanical stimulation. fmn and control flies (male, 2-4 d of age), raised in LD conditions, were collected and placed in glass tubes in activity monitors. The monitor was first maintained in $\mathrm{LD}$, then transferred to DD by wrapping monitors in aluminum foil at the end of the light period. To apply stimuli, monitors were placed on a firm, stable laboratory stone table in a small quiet room. Two small plastic cushions, $7 \mathrm{~mm}$ thick (mild stimuli) or $21 \mathrm{~mm}$ thick (moderate and strong stimuli), were placed under the center of both longitudinal sides of the monitors. Mechanical stimuli were delivered at different times of the subjective night [circadian time (CT) $14,16,18,20,22$ ] by quickly removing both cushions, allowing the monitors to drop onto the stone table. For the mild and moderate stimuli, only one round of stimulation was applied; for strong stimuli, the monitors were dropped consecutively six times.

Activity was monitored every $15 \mathrm{~s}$, and data were used only when there was no activity $5 \mathrm{~min}$ before the stimulus. When a fly began to move within $1 \mathrm{~min}$ after the stimulus and when its movement continued for at least $1 \mathrm{~min}$, the fly was scored as responding to the stimulus; otherwise, it was scored as no response. For flies that responded to a mechanical stimulus, we measured the duration of the response. Once a fly stopped moving and the inactive period continued for at least $5 \mathrm{~min}$, the response was considered to have ended. To calculate response times, data from strong stimulations were used, but similar results were obtained with mild and moderate mechanical stimulation.

Rest (sleep) deprivation. fmn and control flies were placed in glass tubes in an activity monitor, as described in the above stimulation experiments. Manual mechanical stimulation was applied to the monitor at 5 min intervals for the indicated number of hours. The movements of flies were observed by real time monitoring, so that the strength of stimulations could be adjusted to be sufficient to keep all flies awake. Rest deprivations began at CT $16(6 \mathrm{~h})$, CT $18(4 \mathrm{~h})$, and CT $20(2 \mathrm{~h})$ and ended at CT 22. Rest, as defined by 5 min intervals with zero activity, was calculated for each $2 \mathrm{~h}$ period. Rest rebound was calculated as the increase in rest observed in treated flies, relative to nontreated control populations of the same genotype, within a $6 \mathrm{~h}$ period (CT 22-CT 4) after rest deprivation. 
a

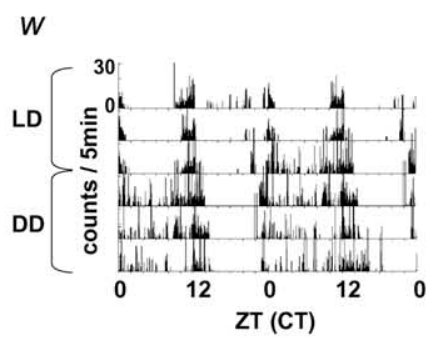

$w ; f m n$
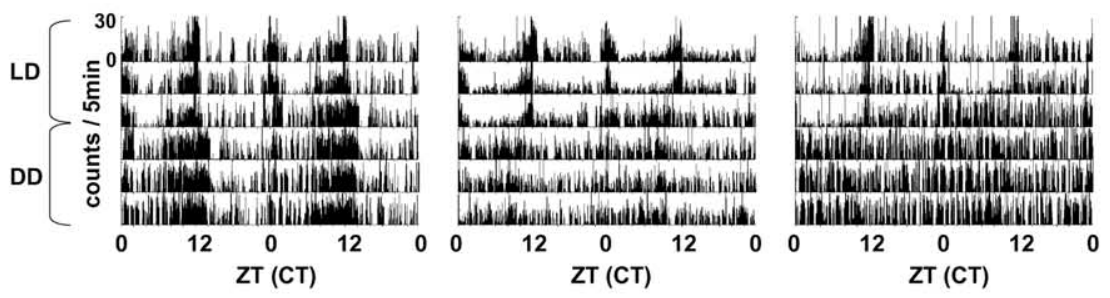

b

W
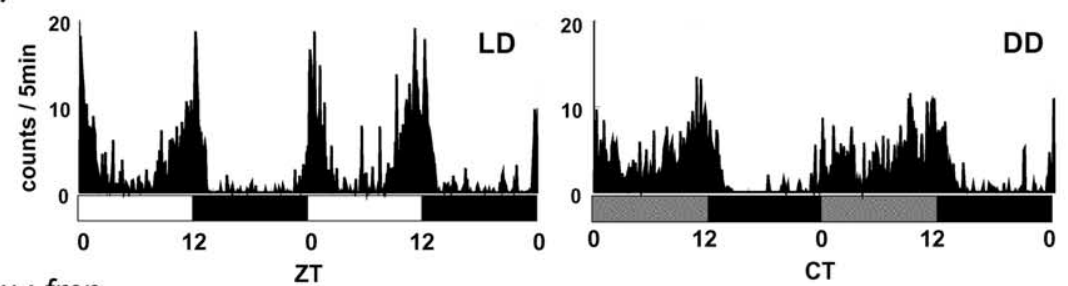

$w ; f m n$
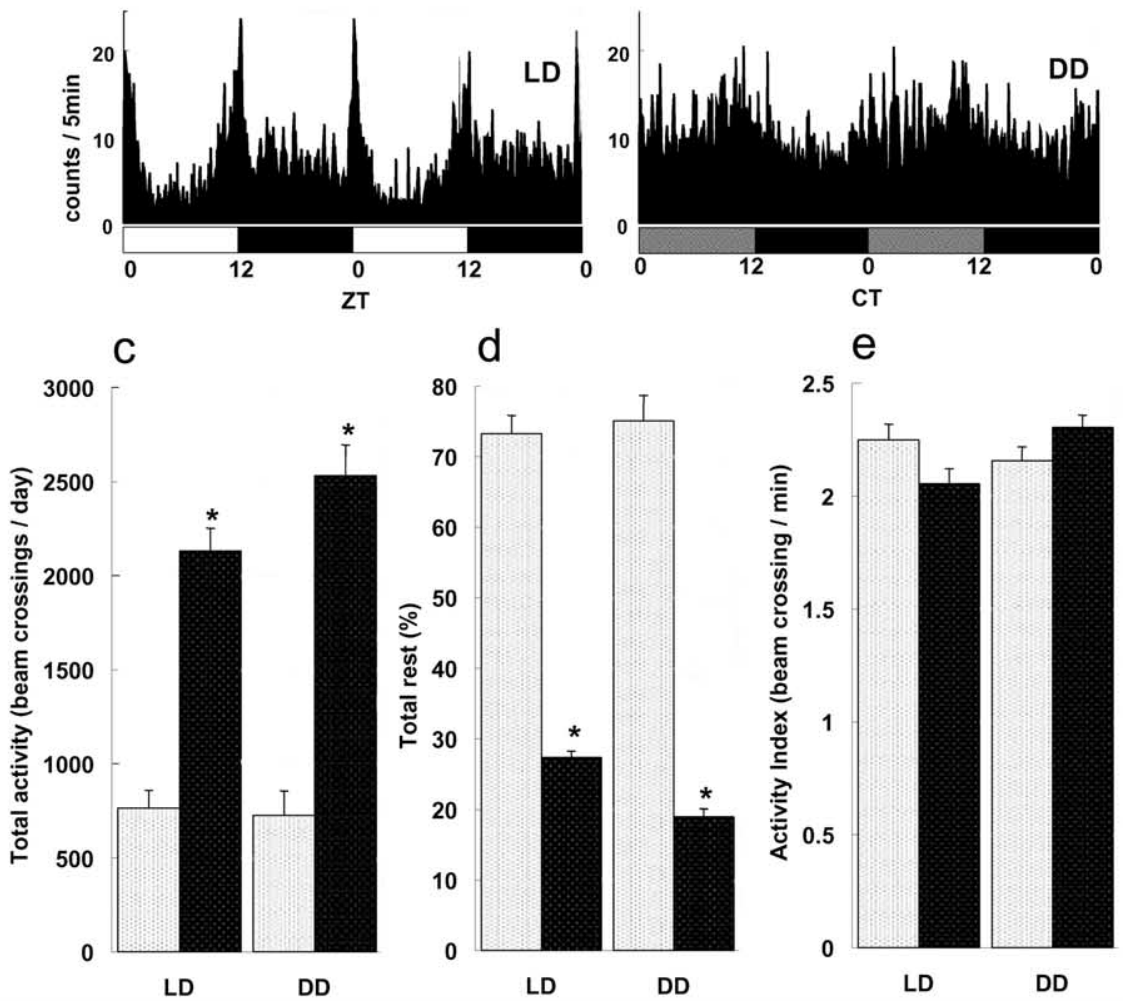

Figure 1. fmn mutants exhibit elevated total daily activity levels, reduced rest, and a normal activity index. $\boldsymbol{a}$, Double plots of locomotor activity data for three representative $w$ and $w$; fmn flies in LD and DD conditions ( $3 \mathrm{~d}$ in LD and $4 \mathrm{~d}$ in DD). Data are plotted as number of activity counts (beam crossings) per 5 min of time. $\boldsymbol{b}$, Population activity averages in LD and DD ( $n=10$ for $w$ and $f(m n)$. c, Total daily activity plots for $w$ and $f m n$ flies. $\boldsymbol{d}$, Average total daily rest for $w$ and $f m n$ populations. $\boldsymbol{e}$, Activity indices for $w$ and fmn flies. Error bars indicate the SEM; * indicates statistically significant differences between $w$ and fmn flies (Student's $t$ test; $p<0.001)$. In $c-e$, gray histograms represent the wild type; black histograms represent fmn.
Determination of life span. In preparation for studies of longevity, $w$ and $w$; fmn male and female flies were separated under $\mathrm{CO}_{2}$ anesthesia and transferred to vials containing fly medium. For each genotype, $\sim 200$ flies were housed in six vials, each containing $\sim 35$ flies. Flies were maintained in a $12 \mathrm{~h}$ light/dark cycle and transferred to new vials twice a week until all flies died. The number of dead flies were counted at each transfer.

\section{Results}

fmn mutants exhibit increased

locomotor activity

In the course of analyzing the nature of sleep-like behavior in Drosophila circadian mutants (Hendricks et al., 2003a), we discovered by serendipity a mutant in a $y$ w; tim $^{01}$ laboratory stock that exhibited high levels of activity at all times of day. This mutant has been named fumin, meaning sleepless in Japanese. We outcrossed fmn mutants to flies from a Cantonized $w^{1118}$ stock (CS10) (Tamura et al., 2003) for seven generations and then examined locomotor activity and rest phenotypes in the descendents. Activity was monitored in individual flies in a $12 \mathrm{~h}$ light/dark cycle and in constant dark conditions (see Materials and Methods for a description of activity recording and the definition of rest/activity parameters). Records for three individual control and fmn flies are shown in Figure $1 a$ ( $3 \mathrm{~d}$ of LD followed by $3 \mathrm{~d}$ of DD). Control $w$ flies displayed a substantial amount of inactivity (5 min intervals without any locomotor activity) both in LD and DD. In contrast, fmn mutants were active almost continuously throughout the day. Although fmn flies displayed high levels of locomotor activity at all times of the cycle, they nonetheless showed rhythmic circadian changes in activity levels for at least the first $2 \mathrm{~d}$ of $\mathrm{DD}$, as shown by the averaged DD records in Figure $1 b$. We note, however, that free-running activity rhythms are not as robust as those seen in the wild type because of the relatively high baseline level of activity in the mutant at all times of subjective day (Fig. 1b). fmn populations show rhythmic, circadian eclosion (data not shown), indicating that fmn is not a clock mutant. Flies in the fmn line show a suppression of activity by light, which is relieved when flies are put into constant conditions (Fig. 1b), but this phenotype may not be caused by fmn, because it is seen in several other lines having a similar genetic background.

On average, fmn mutants showed a twofold to threefold increase in total daily activity in LD and DD compared with control flies (Fig. 1c). In previous studies 


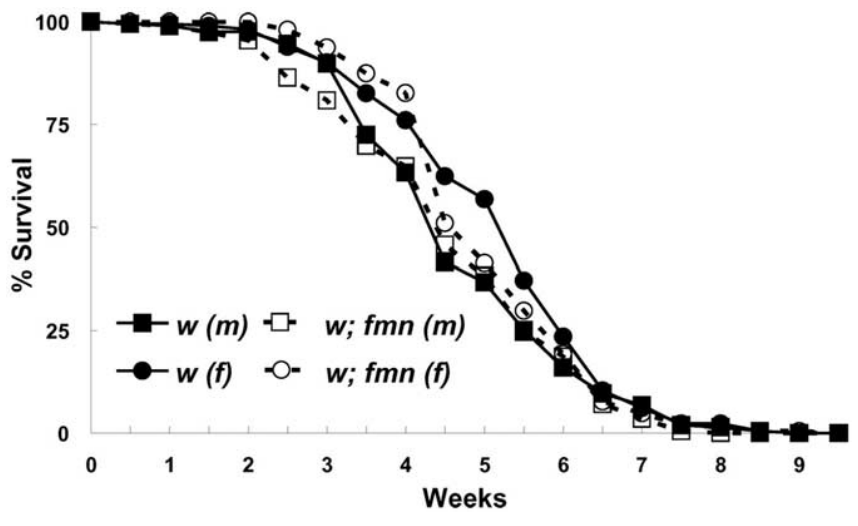

Figure 2. fmn flies have normal life span. Life-span curves are shown for $f m n$ and control flies. Each curve represents $\sim 200$ flies. See Materials and Methods for details of the longevity experiments.

(Hendricks et al., 2000; Shaw et al., 2000), Drosophila activity events were recorded in 10-30 min intervals, and rest (sleep) was defined as those intervals with no activity. In the present study, we used 1 min recording intervals with a moving window analysis package we developed (Hendricks et al., 2003a) and defined rest as a 5 min interval with no activity. Using such a definition, $w \mathrm{fmn}$ flies showed significantly less rest than $w$ controls (Fig. $1 d$ ). Interestingly, however, the activity index, defined as the total daily activity divided by the length of the daily active period, was similar in fmn and control flies (Fig. 1e). This indicates that $f m n$ flies are hyperactive because they have a longer period of daily activity rather than being more active during any given activity period. This increased activity is a result of decreased rest period durations and increased lengths of activity bouts in $\mathrm{fmn}$, both in LD and DD conditions (supplemental Fig. 1, available at www.jneurosci.org as supplemental material and data not shown). The average durations of activity bouts for $\mathrm{fmn}$ and control flies, respectively, were $82.9 \pm 3.0$ and $28.2 \pm 3.0 \mathrm{in}$ LD and $536.4 \pm 271$ and $17.8 \pm 1.4$ in DD.

\section{fmn flies are fertile and have normal longevity}

Sleep is widely believed to be essential for viability, and two different Drosophila sleep mutants have decreased life span (Shaw et al., 2002; Cirelli et al., 2005). Thus, we determined whether fmn affects life span. Those studies indicated that both male and female $\mathrm{fm} n$ flies have normal life span compared with genetic background controls (Fig. 2). Furthermore, the mutant has no obvious morphological, developmental, or fertility phenotypes, although fertility has not been examined quantitatively. Thus, the mutation appears to have a relatively selective effect on activity and rest and no significant impact on development, fertility, or longevity.

\section{Drosophila fmn is an allele of the DAT gene}

Chromosome segregation analysis showed that $f m n$ is an autosomal recessive mutation mapping to the second chromosome. Meiotic mapping, using several dominant genetic markers, showed that fmn is located approximately halfway between Lobe (cytogenetic position 51A4) and Black cell (position 54F6) (see Materials and Materials and Methods). We used recombinant chromosomes carrying fmn and the $L$ marker to further define the position of the behavioral mutation relative to several P-element insertions within the $51 \mathrm{~A}$ to $54 \mathrm{~F}$ interval. The results indicated that $f m n$ mapped near cytogenetic interval 53, close to and to the left of the P-element insertion 12056, which is located in 53C9-10 (Fig. 3a). Perusal of the published Drosophila genome and annotated cDNA sequences (Adams et al., 2000) showed that the Drosophila homolog of the mammalian dopamine transporter $(d D A T)$, a gene for which loss in mice leads to hyperactivity (Giros et al., 1996), resides within region 53C7-8 (Porzgen et al., 2001). Thus, we examined $d D A T$ as a candidate for fmn.

Using a probe representing the whole $\mathrm{dDAT}$ open reading frame, we detected a $3.2 \mathrm{~kb}$ transcript by Northern blot analysis in samples of control $w$ fly head polyA ${ }^{+}$RNA. In contrast, a truncated dDAT mRNA of $1.8 \mathrm{~kb}$ was detected in fmn (Fig. 3b, left). Furthermore, a probe representing the $3^{\prime}$ end of the dDAT ORF did not detect an mRNA in fmn but did hybridize to the $3.2 \mathrm{~kb}$ mRNA in wild-type samples (Fig. 3b, right), indicating that the $3^{\prime}$ end of dDAT is deleted in $f m n$.

To define the nature of the fmn mutation, we cloned dDAT cDNA from $f m n$ flies using the 3' rapid amplification of cDNA end protocol. Sequencing of the fmn dDAT cDNA revealed that there was no splicing at the exon 6 -intron 6 junction, as a result of an insertion of the $5^{\prime}$ portion of a roo transposon (Kaminker et al., 2002) into intron 6. The insertion is located 70 bp downstream of the splice donor junction (Fig. $3 c$ ), and apparently it results in transcriptional termination and polyadenylation of the dDAT mRNA at a site corresponding to sequence within the inserted segment. As a result of an in-frame stop codon in intron 6 , the dDAT protein is truncated at amino acid 343 in $\mathrm{fmn}$, whereas wild-type dDAT is 632 amino acids in length. Southern blot analysis and PCR amplification of genomic DNA showed that the size of the transposon insertion in $f m n$ is $\sim 2 \mathrm{~kb}$ (data not shown), indicating that it is not a full-length $9 \mathrm{~kb}$ roo element.

To verify that the fmn activity phenotype is caused by loss of dDAT activity, we performed phenotypic rescue of $f m n$, using a UAS-dDAT transgene and a pan-neuronal ELAV-GAL4 driver. fmn mutants carrying both ELAV-GAL4 and UAS-dDAT showed a significant but partial phenotypic reversion (i.e., they exhibited decreased daily activity and prolonged sleep periods) (Fig. $3 d, e$, column 4 vs 8 ). Together with the molecular analysis, these results show that $f m n$ is a mutation in the $d D A T$ gene.

\section{fmn mutants exhibit an altered arousal threshold}

Given the decreased rest of fmn flies, we wondered whether arousal might be altered in the mutant. To determine whether fmn flies showed altered arousal threshold, we examined their sensitivity to mechanical stimuli. In these experiments, mechanical shocks were applied to inactive fmn or control flies, during the subjective night, while they were housed within an activity monitor (see Materials and Methods). Positive responses were defined as flies inactive before the stimulus that were activated for at least $1 \mathrm{~min}$ after the stimulus among all flies that were inactive for the $5 \mathrm{~min}$ before the stimulus. As shown in Figure $4 a$, $\mathrm{mm}$ flies were hyper-responsive to mechanical stimuli compared with $w$ control flies, with the greatest difference observed at the weakest stimulus tested. Only 15\% of control flies responded to mild stimuli, but in the same experiments, $49 \%$ of fmn mutants showed a response. A similar differential effect was seen for moderate stimuli (responses by $48 \%$ of control and $88 \%$ of $\mathrm{fmn}$ ), whereas all mutant and control flies responded to strong stimuli. In addition, we measured the durations of the activity bouts induced by mechanical stimulation, and those experiments showed that the responses of $f m n$ flies were lengthened relative to controls. As illustrated by the response times (Fig. $4 b-d$ ), control flies showed stimulus-induced activity with a median bout length of 5 
a
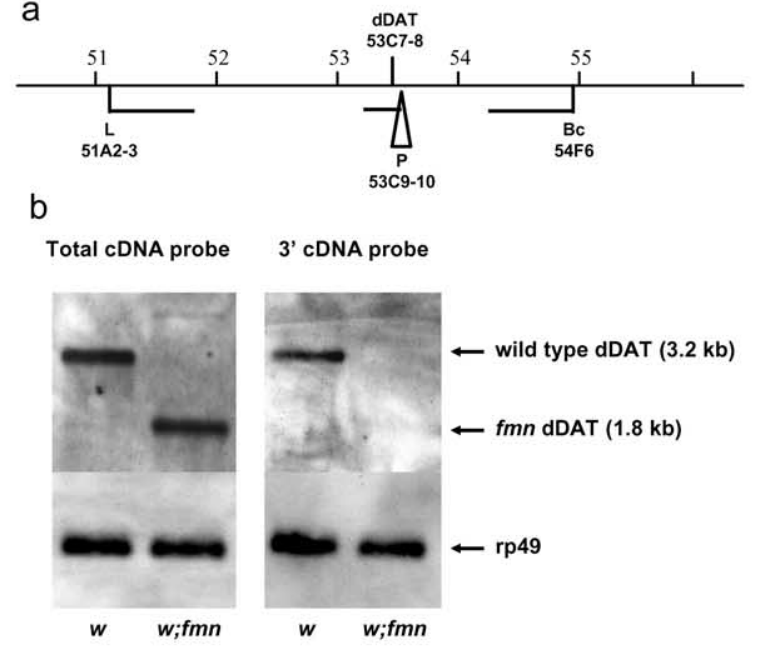

c

wild type
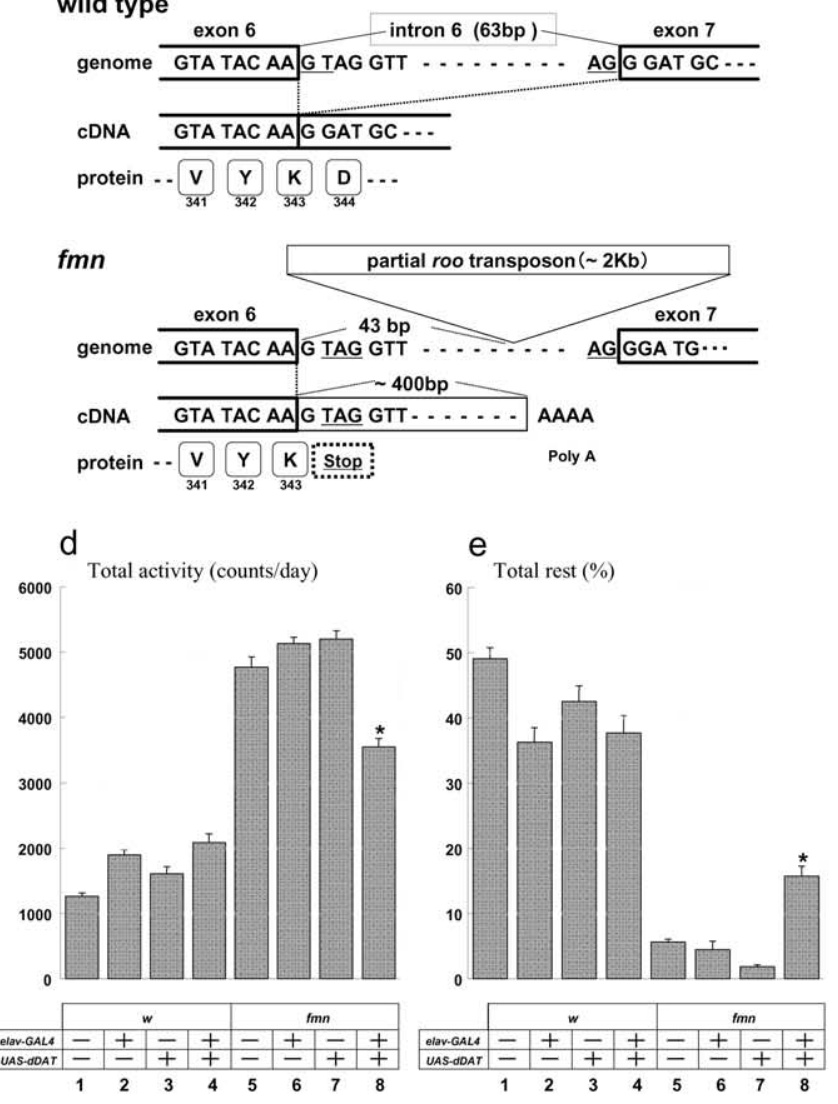

Figure 3. $f m n$ is a dDAT mutation. $\boldsymbol{a}$, Summary of the mapping of $f m n$ by meiotic recombination. The map of the $D D A T$ region is illustrated, showing locations of the dominant genetic markers and P-element insertions used for recombinational mapping of fmn. $\boldsymbol{b}$, Northern blot analysis of $A D A T$ expression. The blot was hybridized with the complete DDAT ORF (left), a $3^{\prime}$ partial ORF (right), or rp49 sequences (positive control). A $3^{\prime}$-deleted, truncated DDAT mRNA is expressed in fmn mutants. See Materials and Methods for details. c, Structures of dDAT genome and CDNA of wild type and fmn. In fmn, exon 6 -intron 6 splicing is affected, presumably because of the heterologous DNA insertion into intron 6 . This aberrant splicing results in the termination of dDAT protein at residue 343 in $\mathrm{fmn}$. $\boldsymbol{d}$, $\boldsymbol{e}$, Rescue of $\mathrm{fmn}$ by neuronal dDAT expression, using elav-Gal 4 as a driver. Quantitative activity and rest analysis was performed for $w$ and fmn mutants using data collected in DD. The presence or absence of ELAV-GALL and the UAS-dDAT responder transgenes are indicated by + or - . The results indicate a partial rescue of the $f m n$ phenotype by transgenic $d D A T$ expression. Differences from nontransgenic and both single transgenic flies $(n=10)$ are statistically significant using Student's $t$ test $\left(^{*} p<0.05\right)$. a
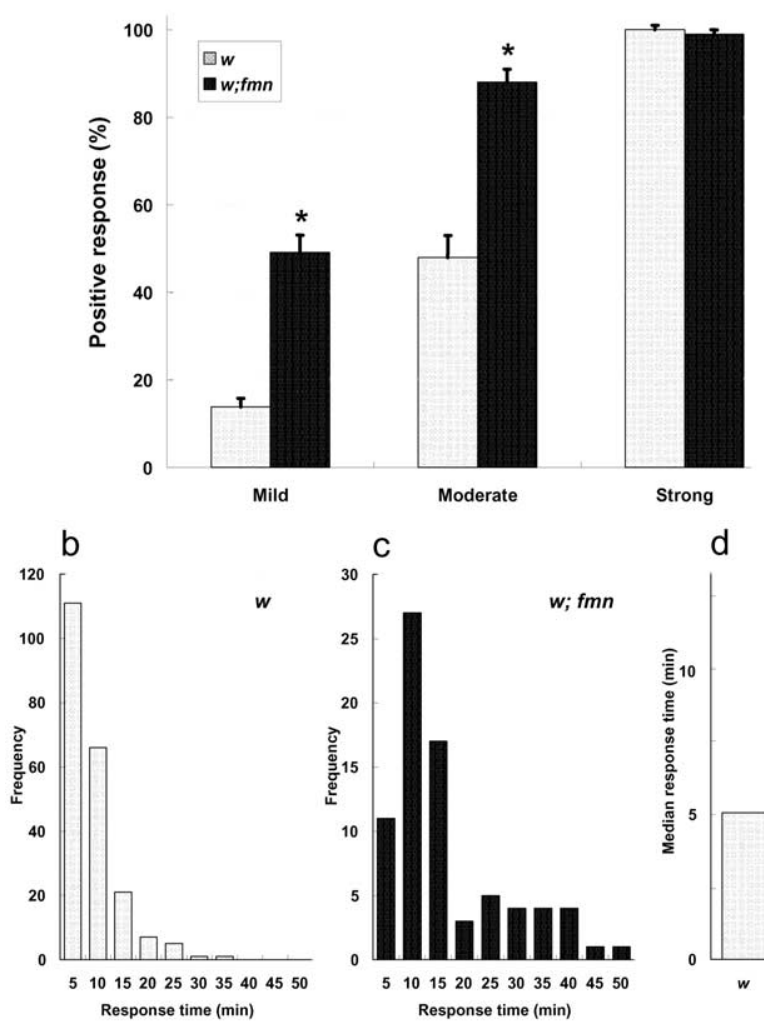

d

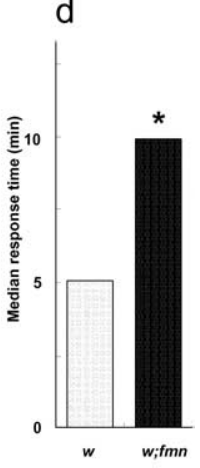

Figure 4. fmn mutants show enhanced responses and prolonged response times to mechanical stimuli. $\boldsymbol{a}$, Responses to mild, moderate, and strong stimuli. fmn mutants showed a larger response than wild-type control flies to both mild and moderate strength stimuli $(n=16$ for both genotypes) $\boldsymbol{b}, \boldsymbol{c}$, Histograms of the distributions of response times in $w$ and fmn flies. Frequency ( $y$-axis) corresponds to the number of stimulated responses of the indicated durations out of the total number of responses. $\boldsymbol{d}$, Average response times for both genotypes (from strong stimulation). fmn mutants show longer bouts of activity after stimulation. Error bars indicate the SEM. * indicates a statistically significant difference between $w$ and $f m n$ flies (Wilcoxon rank-sum test; $p<0.001$ ).

min, whereas fmn mutants remained active for a median time of $10 \mathrm{~min}$, with many flies showing activity periods of $>30 \mathrm{~min}$.

Rest deprivation causes attenuated rebound in fmn mutants Wild-type flies show a prominent rest (sleep) rebound, during which there is more rest after deprivation (Andretic and Shaw, 2005; Ho and Sehgal, 2005). Because the fmn mutation decreased rest, we expected that rebound might also be affected. To address this issue, we examined rebound parameters in control and mutant flies after various durations of rest deprivation. Flies were deprived of rest for 2-6 h during the subjective night by manual mechanical stimulation of the activity monitors housing the flies, and then activity levels were measured after deprivation. The mechanical stimulation was sufficient to completely or nearly completely deprive both $w$ and fmn flies of rest (Fig. $5 a, b$ ). After rest deprivation for 2,4 , or $6 \mathrm{~h}$ during the subjective night, $w$ control flies showed increased rest relative to control nondeprived $w$ flies, in particular at the beginning of the day when flies are normally active (Fig. $5 a$ ). For the wild type, significant differences were observed between treated and control flies with regard to amount of rest for all three durations of deprivation $(2,4$, or $6 \mathrm{~h}$ ), both during and after deprivation at CT 0 , indicating significant rest rebound. Although treated $\mathrm{fmn}$ flies rested significantly less than control flies during deprivation (CT 16, 18, 20), there 
was no significant rebound observed after deprivation at CT 0 or later time points (Fig. 5b). It thus appears that altered synaptic dopamine, as a consequence of lack of dopamine transporter activity, has perturbed the homeostatic regulation of the rest/activity profile in Drosophila.

\section{Discussion \\ fmn, a Drosophila DAT mutant with decreased rest}

This report describes a Drosophila mutant, fumin, that exhibits increased levels of locomotor activity, an alteration of rest (sleep) arousal threshold, and decreased rest rebound in response to deprivation. Although fmn flies exhibit such rest/activity phenotypes, there is apparently no effect of the mutation on development or longevity. This contrasts markedly with the results observed for mutations of two other genes that have been implicated in the regulation of Drosophila rest: $c y c$ and Shaker (Sh). Mutations in cyc or Sh reduce life span, relative to genetic background controls (Shaw et al., 2002; Hendricks et al., 2003a; Cirelli et al., 2005), although complete life-span curves were not reported for $S h$ and there appears to be only a small effect on longevity for the $S h^{102}$ allele (Cirelli et al., 2005). The different effects of these mutations on longevity may reflect the relatively selective effect of $f m n$ on arousal. Alternatively, the effects of $S h$ and $c y c$ mutations on life span might reflect requirements for these genes in developmental or physiological processes other than rest. Cyc protein is a broadly expressed basic helix-loop-helix transcription factor, whereas $\mathrm{Sh}$ is a voltage-activated potassium channel with a broad localization in the nervous system. In contrast, DAT deficits only affect dopaminergic neuromodulation and therefore might have a less general impact on development and physiological processes.

Fmn flies carry a mutation in the Drosophila dopamine transporter gene, indicating that alterations of dopamine signaling are responsible for the observed phenotypes. dDAT functions in the dopaminergic pathway, as shown by (1) dDAT protein has significant sequence similarity to comparable mammalian and invertebrate transporters, (2) $d D A T$ gene expression is restricted to dopaminergic neurons (as expected for a presynaptic transporter), (3) this transporter has a substrate specificity paralleling that of the mammalian DATs, with dopamine and tyramine being the preferred substrates, and (4) the dDAT transporter mediates uptake of dopamine in cell-based assays and responds to dopamine when expressed in Xenopus oocytes (Porzgen et al., 2001).

Dopamine is cleared from the synaptic cleft via presynaptic DAT, and DAT mutant mice exhibit altered presynaptic autoreceptor function, dopamine clearance, and biosynthetic rate (Jones et al., 1998, 1999) in addition to behavioral alterations including spontaneous hyperlocomotion and hyperactivity (Giros et al., 1996; Spielewoy et al., 2000). These phenotypes are presumably caused by the elevated persistence of released dopamine in these mice. Similarly, it seems likely that increased dopamine signaling in fmn is responsible for the observed hyperactivity and shortening of the rest phase, regarded as sleep in Drosophila.

Previous studies in Drosophila implicate biogenic amines in the modulation of activity. In larval Drosophila, 5-HT, OA, TA, and DA regulate locomotion (Yellman et al., 1997; Saraswati et al., 2004) or the sensory-motor circuitry on which such behavior depends (Cooper and Neckameyer, 1999; Dasari and Cooper, 2004). In adult flies, evidence suggests that DA and 5-HT function to regulate locomotor activity and flight, respectively (Pendleton et al., 2002; Banerjee et al., 2004; Lima and Miesenbock, 2005). The study by Lima and Miesenbock (2005) is particularly informative in that it demonstrates state-dependent effects of dopamine neuronal stimulation in behaving flies. In flies with low basal activity, the response to targeted (and transient) stimulation of dopamine neurons is an increased probability of locomotor bouts, whereas a similar stimulation of flies showing high basal activity leads to an inhibition. These results are most consistent with a biphasic role for modulation of locomotor activity by released dopamine, with the highest levels of dopamine release leading to locomotor inhibition. This is in agreement with studies that have examined the responses of flies to the psychostimulant cocaine, an inhibitor of aminergic transporters. Cocaine stimulation of flies results in transient stereotypies and hyperactivity that are strikingly similar to those seen after cocaine exposure to vertebrate animals (McClung and Hirsh, 1998; George et al., 2005). However, the most severely affected flies become akinesic (McClung and Hirsh, 1998; Bainton et al., 2000), consistent with a biphasic effect of high extracellular dopamine.

\section{Arousal threshold and rest rebound are altered by fmn}

Based on the decreased arousal threshold and the prolonged responses of $\mathrm{fmn}$ flies to mechanical stimulation, we suggest that this mutant is characterized by an arousal state with enhanced alertness associated with the expected increase in extracellular dopamine. The absence of significant rest rebound in fmn supports this conclusion, along with our finding that activity level during each arousal period is normal. To our knowledge, this is the first direct evidence that altered arousal threshold and decreased rebound can result from perturbations of dopaminergic signaling. Previous results have indirectly implicated DA in arousal (for review, see Boutrel and Koob, 2004). Those studies 
examined animals with lesions of dopaminergic neuronal populations, changes in firing rates of dopaminergic neurons as a function of sleep states and arousal, or the actions of wake-promoting drugs, some of which modulate DAT or DA receptor activity. The analysis of fmn shows directly that a selective lesion of DAT, presumably with accompanying increased DA levels, results in an alteration of arousal threshold.

A study that came to our attention while this manuscript was in revision used a pharmacological approach to generate findings consistent with our results (Andretic et al., 2005). In that study, flies fed methamphetamine, a drug that interacts strongly with dDAT (Porzgen et al. 2001), showed decreased sleep, with no significant rest rebound. Interestingly, a major effect of methamphetamine is on wake bout duration, similar to our findings with $f m n$.

It is of interest that mouse DAT mutants, like fly fmn, have abnormal sleep (Wisor et al., 2001), although arousal sensitivity has not been explicitly examined. DAT mutant mice show enhanced spontaneous locomotor hyperactivity that is greatly enhanced by the stimulating effects of novel environment (Giros et al., 1996; Gainetdinov et al., 1999). More importantly, Wisor et al. (2001) show that mouse DAT mutants have significantly increased wake bout duration and moderately increased activity levels during the latter half of the active (night) phase of the diurnal cycle. The extension of the active phase in these mice mimics the phenotype of $f m n$ flies that have a lengthened active period and shortened rest phase during the diurnal cycle. DAT knock-out mice also exhibit altered responses to wakepromoting drugs such as GBR12909, modafinil, and caffeine (Wisor et al., 2001). Such mice show increased sensitivity to caffeine and decreased sensitivity to GBR12909 and modafinil, suggesting that the latter two drugs act on DAT to promote wakefulness. Modafinil has wake-promoting properties in Drosophila (Hendricks et al., 2003b), and it will be of interest to determine whether modafinil action is altered in $f m n$ as it is in DAT mutant mice. A role for DAT and dopaminergic signaling in regulating wakefulness in flies and mice provides additional evidence for a similarity between mammalian sleep and insect rest.

Dopamine is probably also important for the regulation of arousal in humans. Parkinson's disease patients, who have reduced dopamine levels, often complain of sleepiness. When treated with L-3,4-dihydroxyphenylalanine (L-DOPA), which increases dopamine levels, they recover from sleepiness, but with excessive L-DOPA, they exhibit insomnia (Clarenbach, 2000; Arnulf et al., 2002). Moreover, patients and animals with narcolepsy, which show increased wakefulness and excessive sleepiness, show a compensational increase in brain $\mathrm{D}_{2}$-like receptors, suggesting a reduced level of dopamine (Okura et al., 2000; Nishino et al., 2001).

\section{fmn flies exhibit circadian activity rhythms}

Although fmn mutants have higher basal levels of activity, they nonetheless exhibit diurnal and circadian rhythms in locomotor activity. However, rhythmicity is less evident compared with that observed in wild-type flies, presumably because there is a higher baseline of activity at all times of day and therefore a corresponding decrease in the amplitude of rhythmicity.

Similar to vertebrates, Drosophila locomotor activity rhythms are controlled by a circadian pacemaker located in the brain (Helfrich-Forster, 1998). The clock system of the fly brain is comprised of $\sim 100$ neurons, including those localized to ventral lateral regions of the protocerebrum, which are critical for the circadian control of locomotor activity. Although it is clear that dopamine has a role in the modulation of insect activity, it is as yet not apparent how the clock system interfaces with dopaminergic output pathways. An understanding of how clock cells communicate with dopaminergic neurons may help illuminate the relationship between circadian and homeostatic sleep regulation. Given the parallels between Drosophila and mammals with regard to circadian control mechanisms (Panda et al., 2002; Reppert and Weaver, 2002; Cyran et al., 2003), the involvement of circadian genes in cocaine responses in flies and vertebrates (Andretic et al., 1999; Abarca et al., 2002), and the parallels reported here between dopamine transporter function in flies and mammals, such studies may also yield insights into the circadian regulation of wakefulness and sleep in mammals.

\section{Precise regulation of DAT may be important for normal dopamine homeostasis}

Part of the evidence that $f m n$ is a mutation in dDAT comes from transgenic rescue using pan-neurally driven expression of dDAT (Fig. 2e). It is notable that this rescue is only partial. One obvious explanation for this partial rescue is that expression of ELAVGAL4 in the dopamine neurons, the sole site of dDAT localization (Porzgen et al., 2001), is weak. However, even stronger dopamine neuron expression of dDAT, under the control of $\mathrm{TH}$ GAL4, yields even weaker rescue (data not shown). This counterintuitive result may indicate that the precise level of dDAT in dopamine neurons is critical for normal dopamine homeostasis. It is possible that homeostatic mechanisms potentially overcompensate for high DAT levels and reduced synaptic DA by hyperactivating postsynaptic receptors, as has been seen after inhibition of dopamine and serotonin neurons (Li et al., 2000). Thus, dDAT may be a component of a precisely regulated dopamine homeostatic mechanism that controls arousal threshold and overall activity levels.

Our study of a Drosophila DAT mutant indicates another striking parallel between Drosophila and vertebrates with regard to the functions of biogenic amine systems. It demonstrates that the regulated reuptake of dopamine by DAT is important for setting arousal threshold. Equally important, the identification of a mutation in the pharmacologically important dopamine transporter opens new avenues for use of this genetically tractable model in pharmacological and behavioral studies.

\section{References}

Abarca C, Albrecht U, Spanagel R (2002) Cocaine sensitization and reward are under the influence of circadian genes and rhythm. Proc Natl Acad Sci USA 99:9026-9030.

Adams MD, Celniker SE, Holt RA, Evans CA, Gocayne JD, Amanatides PG, Scherer SE, Li PW, Hoskins RA, Galle RF, George RA, Lewis SE, Richards S, Ashburner M, Henderson SN, Sutton GG, Wortman JR, Yandell MD, Zhang Q, Chen LX, et al. (2000) The genome sequence of Drosophila melanogaster. Science 287:2185-2195.

Andretic R, Shaw PJ (2005) Essentials of sleep recordings in Drosophila: moving beyond sleep time. Methods Enzymol 393:759-772.

Andretic R, Chaney S, Hirsh J (1999) Circadian genes are required for cocaine sensitization in Drosophila. Science 285:1066-1068.

Andretic R, van Swinderen B, Greenspan RJ (2005) Dopaminergic modulation of arousal in Drosophila. Curr Biol 15:1165-1175.

Arnulf I, Konofal E, Merino-Andreu M, Houeto JL, Mesnage V, Welter ML, Lacomblez L, Golmard JL, Derenne JP, Agid Y (2002) Parkinson's disease and sleepiness: an integral part of PD. Neurology 58:1019-1024.

Bainton RJ, Tsai LT, Singh CM, Moore MS, Neckameyer WS, Heberlein U (2000) Dopamine modulates acute responses to cocaine, nicotine and ethanol in Drosophila. Curr Biol 10:187-194.

Banerjee S, Lee J, Venkatesh K, Wu CF, Hasan G (2004) Loss of flight and associated neuronal rhythmicity in inositol 1,4,5-trisphosphate receptor mutants of Drosophila. J Neurosci 24:7869-7878. 
Borbely AA (1982) A two process model of sleep regulation. Hum Neurobiol 1:195-204.

Boutrel B, Koob GF (2004) What keeps us awake: the neuropharmacology of stimulants and wakefulness-promoting medications. Sleep 27:1181-1194.

Cirelli C, Bushey D, Hill S, Huber R, Kreber R, Ganetzky B, Tononi G (2005) Reduced sleep in Drosophila Shaker mutants. Nat Neurosci 434:1087-1092.

Clarenbach P (2000) Parkinson's disease and sleep. J Neurol 247 [Suppl 4]:20-23.

Cooper RL, Neckameyer WS (1999) Dopaminergic modulation of motor neuron activity and neuromuscular function in Drosophila melanogaster. Comp Biochem Physiol B Biochem Mol Biol 122:199-210.

Cyran SA, Buchsbaum AM, Reddy KL, Lin MC, Glossop NR, Hardin PE, Young MW, Storti RV, Blau J (2003) vrille, Pdp1, and dClock form a second feedback loop in the Drosophila circadian clock. Cell 112:329-341.

Dasari S, Cooper RL (2004) Modulation of sensory-CNS-motor circuits by serotonin, octopamine, and dopamine in semi-intact Drosophila larva. Neurosci Res 48:221-227.

Gainetdinov RR, Wetsel WC, Jones SR, Levin ED, Jaber M, Caron MG (1999) Role of serotonin in the paradoxical calming effect of psychostimulants on hyperactivity. Science 283:397-401.

George R, Lease K, Burnette J, Hirsh J (2005) A “bottom-counting” video system for measuring cocaine-induced behaviors in Drosophila. Methods Enzymol 393:841-851.

Giros B, Jaber M, Jones SR, Wightman RM, Caron MG (1996) Hyperlocomotion and indifference to cocaine and amphetamine in mice lacking the dopamine transporter. Nature 379:606-612.

Helfrich-Forster C (1998) Robust circadian rhythmicity of Drosophila melanogaster requires the presence of lateral neurons: a brain-behavioral study of disconnected mutants. J Comp Physiol [A] 182:435-453.

Hendricks JC, Finn SM, Panckeri KA, Chavkin J, Williams JA, Sehgal A, Pack AI (2000) Rest in Drosophila is a sleep-like state. Neuron 25:129-138.

Hendricks JC, Lu S, Kume K, Yin JC, Yang Z, Sehgal A (2003a) Gender dimorphism in the role of cycle (BMAL1) in rest, rest regulation, and longevity in Drosophila melanogaster. J Biol Rhythms 18:12-25.

Hendricks JC, Kirk D, Panckeri K, Miller MS, Pack AI (2003b) Modafinil maintains waking in the fruit fly Drosophila melanogaster. Sleep 26:139-146.

Ho KS, Sehgal A (2005) Drosophila melanogaster: an insect model for fundamental studies of sleep. Methods Enzymol 393:772-793.

Isaac SO, Berridge CW (2003) Wake-promoting actions of dopamine D1 and D2 receptor stimulation. J Pharmacol Exp Ther 307:386-394.

Jones SR, Gainetdinov RR, Jaber M, Giros B, Wightman RM, Caron MG (1998) Profound neuronal plasticity in response to inactivation of the dopamine transporter. Proc Natl Acad Sci USA 95:4029-4034.

Jones SR, Gainetdinov RR, Hu XT, Cooper DC, Wightman RM, White FJ, Caron MG (1999) Loss of autoreceptor functions in mice lacking the dopamine transporter. Nat Neurosci 2:649-655.

Kaminker JS, Bergman CM, Kronmiller B, Carlson J, Svirskas R, Patel S, Frise E, Wheeler DA, Lewis SE, Rubin GM, Ashburner M, Celniker SE (2002) The transposable elements of the Drosophila melanogaster euchromatin: a genomics perspective. Genome Biol 3:0084.1-0084.20.

Li H, Chaney S, Forte M, Hirsh J (2000) Ectopic G-protein expression in dopamine and serotonin neurons blocks cocaine sensitization in Drosophila melanogaster. Curr Biol 10:211-214.

Lima SQ, Miesenbock G (2005) Remote control of behavior through genetically targeted photostimulation of neurons. Cell 121:141-152.
Marder E, Bucher D (2001) Central pattern generators and the control of rhythmic movements. Curr Biol 11:R986-R996.

McClung C, Hirsh J (1998) Stereotypic behavioral responses to free-base cocaine and the development of behavioral sensitization in Drosophila melanogaster. Curr Biol 8:109-112.

Morales J, Hiesinger PR, Schroeder AJ, Kume K, Verstreken P, Jackson FR, Nelson DL, Hassan BA (2002) Drosophila fragile X protein, DFXR, regulates neuronal morphology and function in the brain. Neuron 34:961-972.

Newby LM, White L, DiBartolomeis SM, Walker BJ, Dowse HB, Ringo JM, Khuda N, Jackson FR (1991) Mutational analysis of the Drosophila miniature-dusky (m-dy) locus: effects on cell size and circadian rhythms. Genetics 128:571-582.

Nishino S, Mao J, Sampathkumaran R, Shelton J (1998) Increased dopaminergic transmission mediates the wake-promoting effects of CNS stimulants. Sleep Res Online 1:49-61.

Nishino S, Fujiki N, Ripley B, Sakurai E, Kato M, Watanabe T, Mignot E, Yanai K (2001) Decreased brain histamine content in hypocretin/orexin receptor-2 mutated narcoleptic dogs. Neurosci Lett 313:125-128.

Okura M, Riehl J, Mignot E, Nishino S (2000) Sulpiride, a D2/D3 blocker, reduces cataplexy but not REM sleep in canine narcolepsy. Neuropsychopharmacology 23:528-538.

Panda S, Hogenesch JB, Kay SA (2002) Circadian rhythms from flies to human. Nature 417:329-335.

Pendleton RG, Rasheed A, Sardina T, Tully T, Hillman R (2002) Effects of tyrosine hydroxylase mutants on locomotor activity in Drosophila: a study in functional genomics. Behav Genet 32:89-94.

Porzgen P, Park SK, Hirsh J, Sonders MS, Amara SG (2001) The antidepressant-sensitive dopamine transporter in Drosophila melanogaster: a primordial carrier for catecholamines. Mol Pharmacol 59:83-95.

Reppert SM, Weaver DR (2002) Coordination of circadian timing in mammals. Nature 418:935-941.

Saraswati S, Fox LE, Soll DR, Wu CF (2004) Tyramine and octopamine have opposite effects on the locomotion of Drosophila larvae. J Neurobiol 58:425-441.

Shaw PJ, Cirelli C, Greenspan RJ, Tononi G (2000) Correlates of sleep and waking in Drosophila melanogaster. Science 287:1834-1837.

Shaw PJ, Tononi G, Greenspan RJ, Robinson DF (2002) Stress response genes protect against lethal effects of sleep deprivation in Drosophila. Nature 417:287-291.

Siegel JM (2004) The neurotransmitters of sleep. J Clin Psychiatry 65 [Suppl 16]:4-7.

Spielewoy C, Roubert C, Hamon M, Nosten-Bertrand M, Betancur C, Giros B (2000) Behavioural disturbances associated with hyperdopaminergia in dopamine-transporter knockout mice. Behav Pharmacol 11:279-290.

Tamura T, Chiang AS, Ito N, Liu HP, Horiuchi J, Tully T, Saitoe M (2003) Aging specifically impairs amnesiac-dependent memory in Drosophila. Neuron 40:1003-1011.

Tobler I (1983) Effect of forced locomotion on the rest-activity cycle of the cockroach. Behav Brain Res 8:351-360.

Vaslet CA, O’Connell P, Izquierdo M, Rosbash M (1980) Isolation and mapping of a cloned ribosomal protein gene of Drosophila melanogaster. Nature 285:674-676

Wisor JP, Nishino S, Sora I, Uhl GH, Mignot E, Edgar DM (2001) Dopaminergic role in stimulant-induced wakefulness. J Neurosci 21:1787-1794.

Yellman C, Tao H, He B, Hirsh J (1997) Conserved and sexually dimorphic behavioral responses to biogenic amines in decapitated Drosophila. Proc Natl Acad Sci USA 94:4131-4136. 